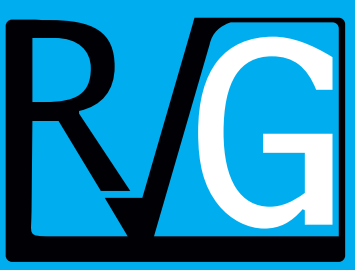

Año 21 No. 74

Abril - Junio 2016

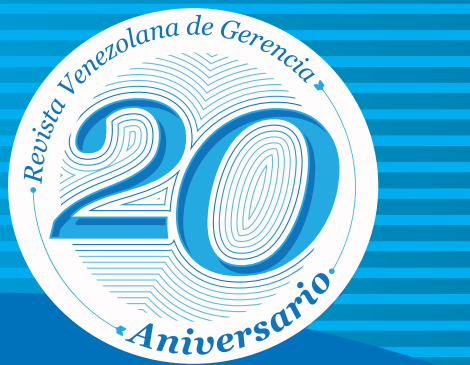

Venezolana de
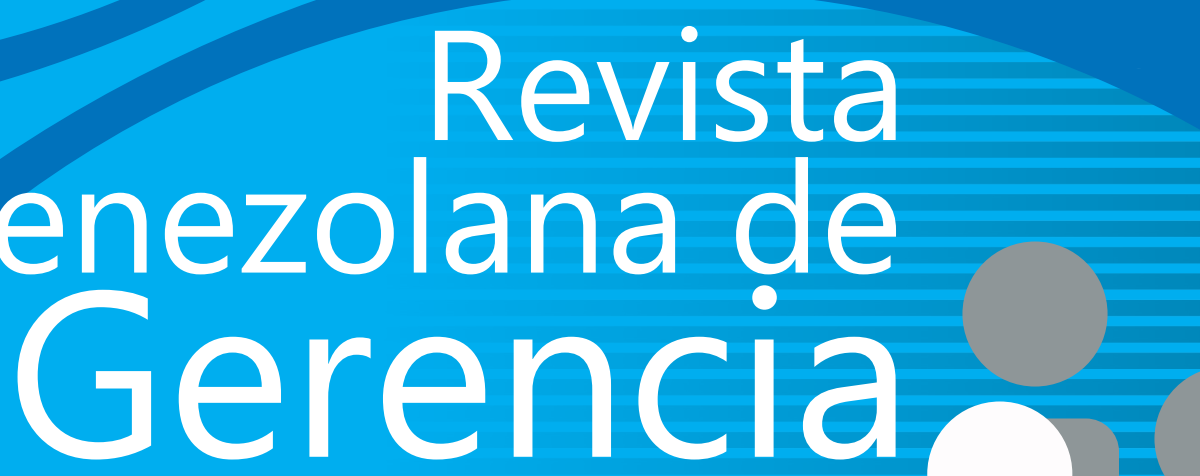


\title{
El financiamiento de la política pública de ciencia y tecnología en Venezuela: avances y desafíos
}

\author{
Finol Romero, Lorayne ${ }^{1}$
}

\begin{abstract}
Resumen
La generación y aplicación de la ciencia como instrumento dirigido a dar respuestas a los problemas de la colectividad, impulsada por un conjunto sistematizado de acciones gubernamentales, conforman la denominada política pública de ciencia, tecnología e innovación; que indiscutiblemente demanda, nuevas fuentes de financiamiento público. A partir de lo cual, esta investigación jurídica documental, plantea analizar exegéticamente el marco normativo de los mecanismos de financiamiento del sistema de ciencia tecnología e innovación en Venezuela. Cuyos resultados evidenciaron avances y retrocesos, siendo el primero de ellos, la institucionalización de la política pública propiamente dicha, a partir la promulgación de la Ley Orgánica en Ciencia Tecnología e Innovación y la Ley del Infogobierno. Cuyo análisis exegético, develó la incorporación al inicio, de un innovador mecanismo de financiamiento parafiscal, cuyas reformas sucesivas de ley le restaron pertinencia, pues se estableció como única modalidad de pago de los aportes, su enteramiento en las cuentas del tesoro. Aparejado de una paulatina disminución de su racionalidad técnica, en virtud de la modificación del ámbito subjetivo.
\end{abstract}

Palabras clave: políticas públicas de ciencia tecnología e innovación; ley orgánica de Ciencia; Tecnología e Innovación; ley infogobierno; obligaciones tributarias parafiscales; fondo parafiscal ciencia y tecnología.

Recibido: 20.03.15 Aceptado: 12.03 .16

1 Abogada Summa Cum Laude. Postgrado en Tributación. Master en Evaluación de Políticas Públicas. Doctora en Derecho. Doctorando en Ciencia política. Prof(a). Del Departamento de Economía y Finanzas Públicas. Jefe de Cátedra. Coordinadora del CIAT. Facultad de Ciencias Jurídicas y Políticas de Luz. Delegada Principal del Consejo de Desarrollo Científico de LUZ. Ioraynefinol@fcjp. luz.edu.ve ; loraynefinol@gmail.com 


\title{
The financing of the politics publishes of science and technology: progress and challenges in Venezuela
}

\begin{abstract}
The generation and application of the science, as an instrument directed for giving answers to the problems of the collectivity, stimulated by a systematized set of governmental actions, shape the public politics of science, technology and innovation, which indisputably demands new sources of public financing. From which, this juridical documentary investigation, considers analyzing exegetically the normative frame of the financing mechanisms of the system of science technology and innovation in Venezuela. Whose results demonstrated advances and setbacks, being the first one of them the institutionalization of the public policies, from the promulgation of the Organic Law in Science Technology and Innovation and the Law of the Infogobierno. Whose exegetic analysis demonstrated the incorporation at the beginning of an innovative mechanism of fiscal financing, whose successive reforms of law reduced relevancy, since it was established as the only payment method of the contributions, its pay in the accounts of the exchequer. Prepared for a gradual decrease of its technical rationality, by the modification of the subjective area.
\end{abstract}

Keywords: public Policies; science technology and innovation; LOCTI; infogobierno; tax debts.

\section{Introducción}

En el mundo de hoy, las personas toman conciencia sobre la aplicación de los adelantos de la ciencia y la tecnología en sus actividades cotidianas, lo que destaca la pertinencia del reconocimiento manifiesto e indiscutible, de reconocer la necesidad de difundir, el papel preponderante que la generación y aplicación de la ciencia y la tecnología reviste en la dinámica social actual, y en consecuencia el rol protagónico que su aplicación representa en la búsqueda de soluciones eficaces y eficientes, dirigidas a atender problemas colectivas impostergables.

En este sentido, el diseño de acciones orquestadas dirigidas a la promoción y creación de capacidades en materia de ciencia, tecnología e innovación con miras al desarrollo sostenible y la erradicación de la pobreza, conforman el mayor desafío que debe afrontar el sector público, que parte de la necesidad de estructurar un conjunto de órganos, estrategias y acciones gubernamentales, concebidas en conjunto como la política pública en ciencia tecnología e innovación.

Ahora bien, este evento de estudio, ha dado paso al diseño de esta investigación jurídica documental, cuyo propósito principal consiste en la consecución del objetivo configurado por el análisis exegético de las leyes que regulan el sistema de ciencia y tecnología en Venezuela, con el propósito de identificar las estructura organizativa del sistema y las obligaciones tributarias que financian la política pública.

En consecuencia, con el propósito de alcanzar el objetivo diseñado, se ha establecido el desarrollo de las 
siguientes categorías conceptuales, en primer lugar se analizan los fundamentos teóricos sobre los cuales se erige la regulación normativa en donde subyace la estructura organizativa del sistema de ciencia tecnología e innovación, en segundo lugar se analiza el marco legal regulatorio necesario para desentrañar el andamiaje legislativo que conforma a la política en ciencia y tecnología, para dar paso al análisis sustantivo de las obligaciones parafiscales reguladas por las leyes de base Ley Orgánica de Ciencia Tecnología e Innovación y ley infogobierno, y por último, se analizan las conclusiones que dan evidencia de los resultados alcanzados.

\section{Uso de la tecnología de información en la Administración pública: algunas consideraciones}

Dentro de los fines esenciales del Estado, se ubica la mejora constante y permanente de las condiciones de vida de las personas, promoviendo la prosperidad y bienestar social. En este sentido, considerando la intervención pública, como un mecanismo que procura, resolver de forma pacífica y consensuada los conflictos de intereses y las dificultades crecientes de los problemas, que la convivencia social genera (Subirats, 2012), es menester del Estado centrarse en la búsqueda permanente de instrumentos dirigidos a mejorar la calidad de la intervención pública, y ello pasa por la necesidad de estrechar las distancias entre las decisiones públicas y las necesidades colectivas de atención prioritaria.

Esta dicotomía, viene aparejada de la tradicional incapacidad burocrática para dar respuestas eficientes y eficaces a las demandas sociales, que amenazan con profundizarse ante los nuevos retos sociales que impone la sociedad del conocimiento tecnológico (Castell, 2005). Frente a esta realidad, converge la necesidad impostergable de diseñar herramientas de gobierno que procuren dar respuesta a los problemas que embargan a nuestra sociedad en la postmodernidad, particularizadas por demandas y requerimientos, cada vez más exigentes.

Ante este escenario, las formas tradicionales de gobierno se hacen ineficaces, y hacen necesario surgir nuevas formas de afrontar el ejercicio de la acción de gobierno, mediante la concreción de dinámicas procedentes de la llamada Nueva Gestión Pública (Subirats, 2012), según la cual, los gobiernos han de profundizar sus redes de conectividad, para mejorar la prestación eficiente y eficaz de los acometidos públicos, abriendo paso a la instauración de nuevas tendencias mundiales, tal y como se desprende de la declaración establecida en documento emitido por el Centro Latinoamericano para el Desarrollo, denominado Carta Iberoamericana Del Gobierno Electrónico, según el cual: "la tecnología de la información es un coadyuvante de la mejora de la gestión pública" (CLAD, 2013: 7).

Es así pues, como el uso de las Tecnología de la información en los órganos de la Administración, actúa como un catalizador que procura la mejora de la información y los servicios ofrecidos a los ciudadanos, orientar la eficacia y eficiencia de la gestión pública e incrementar sustantivamente la transparencia del sector público y la participación de los ciudadanos.

Planteamiento teórico, que ha propiciado que el Estado venezolano, a 
través del Ministerio del Poder Popular con competencia en materia de ciencia, tecnología e innovación conjuntamente con la Comisión Nacional de Tecnologías de Información, impulse la incorporación de esta política de desarrollo sostenible en la Ciencia Y La Tecnología, con el propósito de garantizar la soberanía tecnológica y el desarrollo integral de la nación, mediante el desarrollado de un conjunto de disposiciones legales, que establecen el marco institucional necesario para catapultar la inversión en tecnología de la información en Venezuela, incorporando para ello, un conjunto de obligaciones tributarias dirigidas a la recaudación de los ingresos públicos.

Esta investigación propone un estudio hermenéutico, propio de la Escuela de la Exégesis, dirigido a develar la verdadera voluntad del legislador cuando incorporó en el ordenamiento jurídico positivo vigente, los instrumentos normativos Ley Orgánica en Ciencia y Tecnología (2005) y sus sucesivas reformas del año 2010 y 2014. La Ley de la Administración Pública (2008). Así como, la Ley de Infogobierno. En consecuencia, el contenido ha sido sistematizado, en el orden de las siguientes categorías: Marco legal, obligaciones tributarias para el financiamiento sustentable de la política, y conclusiones.

\section{Marco Legal de la Institucionalización de la política pública en ciencia y tecnología}

En Venezuela el marco legal regulatorio de las políticas en ciencia y tecnología se encuentra configurado por un conjunto de disposiciones, que centran sustrato en la Constitución de la República Bolivariana de Venezuela (1999), en donde se estatuye en el Artículo 110, lo siguiente

"El Estado reconocerá el interés público de la ciencia, la tecnología, el conocimiento, la innovación y sus aplicaciones y los servicios de información necesarios por ser instrumentos fundamentales para el desarrollo económico, social y político del país" (Asamblea Nacional Constituyente, 1999)

En concordancia con el mandato constitucional, se ha desarrollado un conjunto de disposiciones legales, que tiene un común denominador, este es, la institucionalización del uso de la ciencia y la tecnología al servicio de la administración pública en aras de la conformación del gobierno electrónico, así como en la resolución de las necesidades colectivas impostergables de la población, y en ese sentido, puede observarse un extenso conjunto de disposiciones que desarrollan el desiderátum constitucional, y que representan un intento recurrente de alcanzar este objetivo, las cuales de seguidas son analizados sucintamente.

La institucionalización del uso de la ciencia y la tecnología en la función pública está concebida como un sistema dual, que involucra dos aristas; una de ellas, lo concibe como instrumento dirigido a alcanzar el desarrollo sostenible del país, marcada por la generación de ciencia y tecnología, así como su aplicación en la resolución de los problemas de la colectividad, así entendido, como instrumento para alcanzar los fines del Estado.

Y la segunda arista, apunta a la necesidad de su incorporación en la 
gestión pública per se, como herramienta de gobierno dirigida a mejorar la eficacia y la eficiencia de los procesos de toma de decisiones públicas que impactan en la dinámica social cotidiana, verbigracia, la incorporación de las tarjetas inteligentes con interfaces sin contacto, como instrumento de pago del transporte público masivo, son un ejemplo fiel, de la incorporación de la tecnología en procesos administrativos rutinarios.

Ahora bien, en el sentido como ha sido orientada esta investigación, su diseño propone un objetivo preciso, esto es, desde la perspectiva de la escuela exegética, analizar el marco normativo positivo venezolano y los mecanismos de financiamiento que sustentan la institucionalización de la política pública en ciencia y la tecnología, que parte de la identificación de los antecedentes normativos que dieron inicio a su incorporación, así como la estructuración orgánica denominada ámbito subjetivo de la ley, y por último, develar cómo ultima interrogante de investigación, sus mecanismos de financiamiento, esto es, está concebida la inversión para el desarrollo $(i+d)$, queda entendido, que el conjunto de estrategias y objetivos diseñados por la máxima autoridad nacional en ciencia y tecnología, que pretenden alcanzar como resultado de esta política su incorporación en la gestión pública como herramienta de gobierno, denominado Plan Nacional De Desarrollo Económico Y Social De La Nación, forma parte de otro estudio por separado.

En consecuencia, a continuación se propone una análisis normativo de las leyes especiales que dan respuesta a las interrogantes que plantea disipar esta investigación, estas son: Ley Orgánica de Ciencia y Tecnología (2005), su primera reforma del año 2010, la Ley
Orgánica de la Administración Publica (2008), y la ley de Infogobierno (2013).

\subsection{Ley Orgánica de Ciencia tecnología e innovación (LOCTI)}

La promulgación de la primera Ley Orgánica de Ciencia, Tecnología e Innovación (LOCTI) es la materialización del principio constitucional, consagrado en el artículo 110 de la constitución nacional citado up supra; según el cual se declara el interés público del Estado sobre la ciencia, la tecnología y la innovación, así como la aplicación del conocimiento por la sociedad; concebidos como instrumentos indispensables del desarrollo económico y social.

Es así como en fiel cumplimiento al mandato constitucional que atribuye al Estado, personificado por el poder público nacional, la competencia de organización y coordinación general del sistema nacional de ciencia tecnología e información; sin lugar a dudas, se sientan las bases estructurales de la Política Pública de Ciencia y Tecnología en Venezuela.

Es pues, con la entrada en vigencia de la Ley Orgánica de Ciencia, Tecnología e Innovación (LOCTI); a partir del 03 de agosto de 2005 (Gaceta Oficial de la República Bolivariana de Venezuela No. 38.242), como se dio inicio al proceso de institucionalización de la ciencia y la tecnología, concebidas como herramientas dinamizadoras del ejercicio de la actividad científica en el país en fomento de la capacidad para la generación, uso y circulación del conocimiento.

La obligación configurada en la LOCTI (2005), se estructuraba sobre la base de la consecución de una serie objetivos de la llamada corriente extra fiscal, por cuanto, el creador originario 
de este instrumento dio paso a la configuración de un mecanismo tributario, que no solo persigue arbitrar recursos para el financiamiento del gasto público que representa la promoción de la actividad científica, sino que adicionalmente, incorporó otros mecanismos de cumplimiento voluntario de la obligación pecuniaria, con fines extrafiscales; dirigidos a impulsar la estructuración de una red de intervinculación entre la comunidad científica, sociedad y el estado, incorporando a la autogestión de los aportes de iniciativa privada, en beneficios propios de la empresa como aportante del sistema, y/o de las demás entidades consideradas sujetos de aplicación de la ley, evidentemente bajo la coordinación y control del marco de regulación establecido, y en cumplimiento de su corresponsabilidad social.

Este instrumento fiscal, se corresponde claramente con los innovadores mecanismos de política fiscal tributaria diversificado y de acción directa en la estabilización macroeconómica (Musgrave, 1993), que abrió paso a la incorporación de vinculo jurídico de carácter prestacional, de naturaleza y esencia pecuniaria tributaria con fines extrafiscales, atinentes al desarrollo de una determinada política pública de estímulo a la generación de productos de la actividad científica, encaminada a alcanzar el firme propósito establecido en la Constitución Nacional, esto es la "elevación del nivel de vida de la población y la protección de la economía nacional" (Asamblea Nacional Constituyente, 1999).

\section{Estructura Del Sistema de Ciencia Tecnología}

Los elementos que componen al Sistema de Ciencia y Tecnología, están plenamente identificados en las leyes especiales que institucionalizan su incorporación en el ordenamiento jurídico positivo vigente. En ese sentido, a continuación se procede a identificar a cada uno de ellos.

\section{- Sujetos de derecho que forman parte del sistema nacional de ciencia y tecnología}

Entendidos como los organismos públicos o privados adscritos al sistema nacional de ciencia y tecnología, regulados en el artículo 3, y en conjunto fungen como actores políticos propulsores del sistema. Los cuales quedaron establecidos en las siguientes categorías:

\section{- Ministerio del Poder Popular para Ciencia y Tecnología}

Es el órgano rector del sistema nacional de ciencia tecnología e innovación. Dentro de sus atribuciones destaca la competencia para coordinar y articular las políticas y estrategias que rigen la actividad científica, tecnológica, de innovación y sus aplicaciones.

\section{- FONACIT}

El Fondo Nacional de Ciencia, Tecnología e Innovación, FONACIT, es un Instituto Autónomo con personalidad jurídica y patrimonio propio, adscrito al ministerio de ciencia y tecnología, que goza de las prerrogativas, privilegios y exenciones de orden procesal, civil y tributario conferidos por la normativa aplicable a la República. El Objeto del Fondo Nacional de Ciencia, Tecnología e Innovación consiste en brindar apoyo financiero para la ejecución de los programas y proyectos definidos por el ministerio con competencia en materia de ciencia y tecnología, a través del Plan Nacional de Ciencia, Tecnología e Innovación, según las modalidades de financiamientos previstos en el presente Reglamento. 


\section{- Observatorio Nacional de Ciencia, Tecnología e Innovación (oncti)}

Es un Organismo creado para apoyar la gestión del conocimiento y el seguimiento del Sistema Nacional de Ciencia, Tecnología e Innovación. Entre sus objetivos se encuentran:

1.- Propiciar estrategias que conviertan la información en oportunidad para fortalecer el Sistema Nacional de Ciencia, Tecnología e Innovación (SNCTI).

2.- Crear registro de los integrantes del SNCTI.

3.- Búsqueda, detección y seguimiento de la información con dicho sistema y el análisis del entorno.

El ministerio con competencia en materia de ciencia y tecnología, a través del Observatorio Nacional de Ciencia Tecnología e Innovación ejercerá las atribuciones conferidas en la Ley Orgánica de Ciencia, Tecnología e Innovación en lo que concierne a las facultades contempladas en los Títulos III y IV de la Ley. Así como las contempladas en el Título VIII cuando éstas versen sobre el incumplimiento de las obligaciones referidas a los aportes e inversiones.

\section{- Organismos adscritos}

Son sujetos de derecho público o privado, con facultad legislativa de desarrollas actividades de ciencia y tecnología con fondos dependientes del Ministerio con competencia en materia de Ciencia y Tecnología, integrantes del Sistema Nacional de Ciencia, Tecnología e Innovación, que comercialicen propiedad intelectual de bienes y servicios, desarrollada con recursos provenientes parcial o totalmente de los financiamientos otorgados a través del Ministerio con competencia en materia de Ciencia y Tecnología o sus organismos adscritos conforme al artículo 34 de la Ley Orgánica de Ciencia, Tecnología e Innovación.

\section{- Beneficiarios}

En coherencia con los presupuestos factuales que propiciaron la promulgación de la ley, se introduce normativamente la conformación de la Red Nacional de Actores, que en forma organizada y bajo la coordinación del Ministerio de Ciencia y Tecnología, configuran el ámbito subjetivo, del denominado Sistema Nacional de Ciencia y Tecnología e Innovación, los cuales se detallan a continuación.

\section{a) La Empresa Obligada}

La ley permite que la empresa obligada a realizar la inversión y/o el aporte, a su vez sea beneficiaria; en el entendido que podrá invertir en proyectos de innovación relacionados con las actividades de la empresa misma (Art. 42; Numeral 4 LOCTI); en consecuencia el monto al que está obligado a invertir/ aportar en proyectos de investigación podrán ser ejecutados en si misma; siempre y cuando cumpla con los supuestos establecidos en la norma y sean efectivamente pagados durante el ejercicio económico anual al que corresponda cumplir con la obligación.

\section{b) Los organismos adscritos al Ministerio}

Se corresponde con todos los FUNDACITE creados en las regiones del país, los cuales atienden a la porción de la dimensión geográfica a la cual esta circunscritos. Entre ellos se encuentran; Fundacite Zulia, Fundacite Lara, Fundacite Anzoátegui, Fundacite Carabobo, 
Fundacite Aragua, Fundacite Falcón, Fundacite Monagas, Fundacite Bolívar, Fundacite Mérida, Fundacite Táchira, Fundacite Barinas, Fundacite Amazonas, Fundacite Portuguesa, Fundacite Sucre.

c) Las universidades Públicas

d) Universidades Privadas

e) Unidades, Institutos o centros de investigación y desarrollo

f) Cualquier otra institución pública $o$ privada que realice actividades de ciencia y tecnología

Queda entendido, que el espíritu propósito y razón del legislador en el año 2005, cuando se promulgó el texto de ley, era crear una red nacional conformada por un conjunto de actores, que en forma organizada y bajo la coordinación del Ministerio de Ciencia y Tecnología, conformaban el denominado Sistema Nacional de Ciencia y Tecnología e Innovación, actores políticos encargados de propiciar la investigación, la tecnología y su aplicación en el marco de un Plan Nacional De Desarrollo Nacional, ahora bien, los presupuestos de hecho antes narrados perdieron su vigencia, a propósito de la reforma de ley experimentada vía reforma de ley, impulsada por la Asamblea Nacional en el año 2010, analizada a continuación.

\subsection{La Ley de Reforma de la Ley Orgánica de Ciencia, Tecnología e Innovación (2010)}

Esta ley fue publicada en Gaceta Oficial No 39.575 de fecha 16 de diciembre de 2010. Esta ley, persigue el mismo propósito inicial, de utilizar la actividad científica del país; como instrumento indispensable para el desarrollo económico y social. No obstante, esta incorpora otra modalidad de cumplimiento de la obligación parafiscal, en donde, se reformó el mecanismo de pago, incorporando como único mecanismo de pago de esta obligación parafiscal lo establecido en el Articulo 24, según el cual:

Artículo 24: "El Fondo Nacional
para la Ciencia la Tecnología
y la Innovación (fonacit), ente
adscrito a la autoridad nacional
con competencia en materia de
ciencia, tecnología, innovación y
sus aplicaciones, es el responsable
de la administración recaudación
control, verificación y determinación
cuantitativa y cualitativa de los
aportes para la Ciencia, Tecnología,
Innovación y su aplicaciones. La
fiscalización compete a la autoridad
nacional en forma directa". (LOCTI,
2010).

Queda prescrito, que el aporte de la obligación prestacional pecuniaria establecida en la Ley, solo puede ser dirigido al fondo de ciencia tecnología e innovación (fonacit), restándole espacio a la inversión directa de la ciencia y la tecnología autogestionada, con aporte de los sujetos obligados de la ley, así como introspectivamente desde las grandes empresas obligadas, lo que ha significado un retroceso importante para la autonomía financiera de la actividad científica del país.

Se elimina todo vestigio del sistema hibrido de ciencia y tecnología, conformado por una serie de actores publico privados, encargados de motorizar las actividades de ciencia y tecnología del país, y aquellos que obligados-beneficiarios que utilizaban 
los aportes como inversiones dentro de sus propias organizaciones para formar el recurso humano, bien sea como becas para posgrados, cursos de formación orientados a mejorar la productividad de los trabajadores dentro de las organizaciones, quedó excluida totalmente.

El ámbito subjetivo de la ley, reformada en el año 2010, quedó reducido a lo establecido a continuación:

1. La autoridad nacional con competencia en materia de ciencia, tecnología, innovación y sus aplicaciones, sus órganos y entes adscritos.

2. Todas las instituciones, personas naturales y jurídicas que generen, desarrollen y transfieran conocimientos científicos, tecnológicos, de innovación y sus aplicaciones.

3. Los ministerios del Poder Popular que comparten, con la autoridad nacional con competencia en materia de ciencia, tecnología, innovación y sus aplicaciones, la construcción de las condiciones sociales, científicas y tecnológicas para la implementación del Plan Nacional de Desarrollo Económico y Social de la Nación.

4. Las comunas que realicen actividades de ciencia, tecnología, innovación y sus aplicaciones.

Esta disposición normativa, junto a lo establecido en el ámbito referido al destino de los fondos, en el art. 23 y 24 , cierran toda posibilidad a la autogestión del conocimiento como iniciativa privada, quedando reducido al criterio que la autoridad competente, llámese Fondo Nacional para la ciencia la tecnología y la innovación (FONACIT), considere estén en coherencia con el Plan Nacional, en cuyo ejercicio reposa la competencia de administración, recaudación, control y verificación de los aportes para la ciencia y la tecnología.

Basta un simple análisis exegético, de los artículos 34 al 44 de la LOCTI (2005), comparativamente con los 7 artículos, del mismo Título III referido a los aportes de la LOCTI (2010), para deducir que el espíritu, propósito y razón del legislador que creó el sistema en el año 2010, cambió significativamente, con una marcada tendencia a la fiscalidad de los fondos públicos, administrados centralizadamente por el organismo con competencia en todo el territorio nacional.

\subsection{La Ley de Reforma de la Ley Orgánica de Ciencia, Tecnología e Innovación (2014)}

En fecha 18 de noviembre de 2014 fue publicado en la Gaceta Oficial de la República Bolivariana de Venezuela No. 6.151 Extraordinario, el Decreto con Rango, Valor y Fuerza de la Ley de Reforma de la Ley Orgánica de Ciencia, Tecnología e Innovación.

Esta reforma estuvo orientada a esclarecer los criterios de determinación y cuantificación de la obligación tributaria, toda vez, la desnaturalización que experimentó el aporte a la ciencia y la tecnología en la Reforma del 2010, y en ese sentido en esta oportunidad el legislador, puntualizó aspectos referidos específicamente a la base imponible; periodo imponible, así como las consecuencia jurídicas que se desprende del incumplimiento de pago de las obligaciones, la incorporación de los agentes de retención como sujetos 
pasivos de la relación jurídica tributaria, y la reorganización del Fondo Nacional de Ciencia y Tecnología, como actor central del sistema, pues le fu conferida la competencia de recaudación, administración y fiscalización de los aportes.

\subsection{Ley Orgánica de la Administración Pública}

Esta ley establece los principios y bases que rigen la organización y el funcionamiento de la Administración Pública; y establece las normas básicas sobre los archivos y registros públicos, fue publicada en Gaceta Oficial No 5.890 de fecha 15 de julio de 2008. La promulgación de esta reforma, se verifica en un entorno político y social económico, impulsado por el desiderátum del máximo tribunal en sala Constitucional conocido como la Sentencia del Tribunal supremo de Justicia en Sala Constitucional, en fecha 15 de Abril de 2008, donde se exhorta a la Asamblea Nacional a realizar un conjunto de reformas legales para ajustarse al nuevo criterio de concentración de la función pública de la Administración, basado en una modelo de descentralización cooperativa o funcional, entre los cuales se incluyeron a los denominados Consejos Comunales, como organización comunitaria con competencia para la administración de recursos públicos, y se les supeditó al cumplimiento de todos los principios rectores como al resto de los órganos y entes públicos, y muy específicamente al cumplimiento del principio de eficiencia en la asignación y utilización de los recursos públicos.

Lo que ha ocasionado, el aminoramiento paulatino de las competencias de los entes político territoriales de los órganos que configuran al poder público descentralizado, quienes cada vez, resultan más disminuidos en el ejercicio de sus competencias constitucionales, toda vez, que desde la iniciación del proceso experimentado desde 2008, es posible la reversión del ejercicio de sus competencias a la administración centralizada.

Esta Ley, incorpora en la Administración Pública en Venezuela una acentuada tendencia al control de la gestión pública a nivel central, supeditando esta competencia a dos organismos públicos, que ejercen tareas de evaluación de desempeño, y estos son: 1) la Comisión Central de Planificación y 2) las Oficinas Estratégicas De Evaluación.

\subsection{Ley del Infogobierno}

Esta Ley es la más reciente del conjunto de disposiciones que regulan esta materia, cuyo objeto de regulación es establecer los principios, bases y lineamientos que rigen el uso de las tecnologías de información en el Poder Público y el Poder Popular, para mejorar la gestión pública y los servicios que se prestan a las personas; impulsando la transparencia del sector público; la participación y el ejercicio pleno del derecho de soberanía; así como, promover el desarrollo de las tecnologías de información libres en el Estado; garantizar la independencia tecnológica; la apropiación social del conocimiento; así como la seguridad y defensa de la Nación. Fue promulgada en Gaceta Oficial № 40.274 de fecha 17 de octubre de 2013 con una vacatio legis de 10 meses que se venció el 17 de agosto de 2014.

El contenido de esta ley, está dirigido a establecer los lineamientos que regirán y facilitarán el uso de las 
tecnologías de información entre el Poder Público y el Poder Popular, estableciéndose las condiciones necesarias para fomentar la mejora permanente de los servicios que se prestan a los particulares.

Los órganos de la administración Pública que ejercen las competencias de la ley, se les reconoce como Organismos Públicos con competencia en tecnologías de información, tal y como a continuación se detalla:

\section{a) El Consejo Nacional para el Uso de las Tecnologías de Información en el Poder Público, (CNTI).}

Es el ente que asesorará al Poder Público en los asuntos relacionados con las tecnologías de información, estableciendo las políticas y estrategias para su adecuado acceso, desarrollo, uso y aprovechamiento.

b) La Comisión Nacional de las Tecnologías de Información:

Es el órgano que asumirá las competencias de ley en esta materia, bajo la figura jurídica de instituto público (Art. 40 Ley Infogobierno) con competencias financieras, administrativas, presupuestarias, técnicas, normativas y de gestión de recursos, al que corresponderá, entre otros, la elaboración del Plan Nacional de Tecnologías de Información para el Estado.

c) Unidades de Apoyo.

d) Subsistemas del sistema nacional de protección y seguridad informática (Superintendencia de Servicios de Certificación Electrónica), quien tendrá dentro de sus competencias:

En este sentido, el legislador nacional dispuso en los artículos 58 y 59 , las competencias que corresponden a esta Superintendencia, entre las cuales se encuentra, la aprobación, certificación y homologación de los equipos o aplicaciones criptográficas, con el objeto de garantizar la integridad, calidad e independencia tecnológica, aprueba, certifica y homologa los equipos o aplicaciones con soporte criptográfico que use el Poder Público y el Poder Popular.

Los registros públicos de homologación, estarán cargo de La Superintendencia de Servicios de Certificación Electrónica, quien es el órgano encargado de supervisar y exigir los certificados de homologación o sellos de certificación por modelo o versión de los equipos o aplicación con soporte criptográfico. A tal efecto, lleva un registro público del código de homologación para proveedores de servicios de certificación de los entes u organismos del Poder Público y del Poder Popular que hayan sido homologados y certificados.

\section{Obligaciones parafiscales como mecanismos de financiamiento de la ciencia y tecnología en Venezuela}

Entendiendo como categoría conceptual, obligación tributaria parafiscal, al vínculo jurídico prestacional de carácter coercitivo y obligatorio, cuyas percepciones dinerarias no ingresan al sistema de tesorería de la administración financiera del sector público, que en otrora, bajo el imperio de la Ley de Hacienda Pública de 1971, se denominaba fisco, hoy sistema nacional de tesorería.

El destino de estas percepciones dinerarias, son los patrimonios autónomos de los organismos recaudadores y administradores de los fondos, pero igualmente son detracciones 
económicas exigidas mediante ley, creadas por el Estado en ejercicio de su poder de imperio con ánimos de garantizar el cumplimiento de sus fines, en cuyo caso, conforman obligaciones parafiscales objeto de estudio de esta investigación.

\subsection{Ley Orgánica De Ciencia Tecnología E Innovación (Locti)}

El tributo que contrae la LOCTI (2005), feu concebido como una obligación legal, en cuyo contenido subyace, una pprestación susceptible de valoración en dinero, que el Estado exige en ejercicio de su poder de imperio en virtud de una Ley y para cubrir los gastos que le demande en cumplimiento de sus funciones (Villegas, 1999), no es fácilmente tipificable mediante el criterio de clasificación clásico, Impuestos, Tasas y Contribuciones. Pues, responde al desarrollo de nuevos criterios clasificatorio según el cual, el Estado puede dar paso a la configuración de ciertas obligaciones legales de carácter prestacional económico, a cargo de específicos entes públicos; con ánimos de garantizar su financiamiento autónomo, tal es el caso de la Obligaciones Prestacionales - Exacciones Pecuniarias de carácter Parafiscal (TSJ, 2006).

Siendo el objeto de esta ley, el desarrollo del sistema nacional de ciencia tecnología e innovación, exige la incorporación de un instrumento fiscal dirigido a alcanzar tal propósito, este es; la obligación legal de carácter prestacional tributario denominada aporte LOCTI, tal y como se analiza a continuación los elementos constitutivos de la obligación legal tributaria. a) Sujetos Obligados: Aquellas personas de derecho, sobre las cuales recae la obligación de aportar o invertir en actividades de ciencia, tecnología e innovación. Establece el legislador, en el artículo 44 de la Locti; la definición de grandes empresas; entendidas como aquellas que perciban ingresos brutos anuales superiores a 100.000 U.T.; dentro de las cuales se encuentran las que señala el dispositivo legal en forma taxativa, Compañías anónimas y sociedades de responsabilidad limitada, Sociedades en nombre colectivo, Asociaciones, fundaciones, corporaciones y demás entidades jurídicas y económicas, Establecimientos permanentes, centros o bases fijas situados en el territorio nacional.

\section{b) Contenido De La Obligación}

Tributaria: Las empresas calificadas como obligadas, por el registro correspondiente de certificación de aportante otorgado por FONACIT, según su estimación de ingresos brutos en el año base en su última declaración ISLR; tienen todo un año calendario siguiente, al surgimiento de la obligación de realizar su aporte a la ciencia y la tecnología, al Ministerio del Poder Popular para Ciencia y Tecnología. Cuyo incumplimiento es sancionado es de $50 \%$ del monto a aportar.

En consecuencia, esta ley, obliga a las empresas públicas y privadas a realizar un aporte al Estado al finalizar su ejercicio fiscal, a cargo del Fondo Nacional de Ciencia y Tecnología, en procura del fomento de las actividades de investigación, apropiación social del conocimiento, transferencia e innovación tecnológica en el país.

Esta obligación de contenido pecuniario, estatuida en la ley, ha sido 
diseñada como uno de los instrumentos innovadores del sistema heterodoxo tributario venezolano (Finol,2009); según el cual, los sujetos obligados por la ley, deben cumplir con la obligación de aportar al Fonacit; y por eso se consideran herramientas fiscales con fines estabilizadores; que no solo persiguen fiscales (recaudatorios), sino fines extrafiscales; esto es, propulsar la actividad científica del país; como instrumento indispensable para el desarrollo económico y social.

\subsection{Ley Infogobierno}

Este instrumento normativo, regula todo lo relativo al uso por parte del Estado de la tecnología y los sistemas de información, por eso su denominación Infogobierno, sin embargo, el legislador nacional, incluyó una serie de materias, entre ellos la simplificación de los procedimientos ante la Administración Pública, y la creación de tributos parafiscales, así como protección de datos personales (Araque Reyna,2013).

Con relación a las obligaciones tributarias, en el capítulo I, del título III, el legislador contempló dos (2) contribuciones parafiscales, cuyas prestaciones pecuniarias sustantivas se pagarán a la Comisión Nacional de Tecnologías de Información y se deducirán del pago del Impuesto Sobre la Renta (IsIr), así como tres tasas y una contribución especial a favor del Fondo Nacional de Ciencia Tecnología e Innovación, como se especifican a continuación a partir del supuesto factico tipificado por el legislador como generador de la obligación:

a) Actividades de importación, distribución y comercialización de software privativo al poder público

Las personas jurídicas que se dediquen a la importación, distribución y comercialización de software privativo al
Poder Público, pagarán el dos y medio por ciento $(2,5 \%)$, de la utilidad neta del ejercicio.

b) Prestación de servicios de software privativo al poder público

Toda persona que preste servicios de software privativos al Poder Público, pagará una contribución del uno y medio por ciento $(1,5 \%)$ de la utilidad neta del ejercicio, dentro de los noventa (90) días siguientes al cierre del ejercicio fiscal.

En la Ley, se estatuyen las Tasas y contribuciones especiales, dentro de las cuales la ley del infogobierno, establece tres (03) tasas, y una contribución especial en CTI, a favor del fondo de ciencia tecnología e innovación, tal y como se detalla a continuación:

a) Certificación de cumplimiento (Artículo 62)

El Poder Público debe solicitar ante la Comisión Nacional de las Tecnologías de Información, la certificación del cumplimiento de las disposiciones de la presente Ley y demás normativa aplicable de los programas informáticos por equipos de computación según su tipo o modelo, el cual causa una tasa de cincuenta Unidades Tributarias (50 U.T.).

b) De las tasas por certificación y homologación de los equipos - aplicaciones con soporte Criptográfico (Artículo 63)

La homologación de los equipos o aplicaciones con soporte criptográfico, a que hace mención el artículo 58, tendrá una duración de tres años y su solicitud de tramitación causará una tasa de trescientas Unidades Tributarias (300 U.T.). Las aplicaciones y equipos con soporte criptográfico libre estará $n$ exentos del pago de la tasa prevista en el presente artículo. 
c) De las contribuciones especiales por la utilización de software privativo (Artículo 67)

El órgano o ente del Poder Público al igual que el Poder Popular que sea autorizado a adquirir, usar y actualizar un software privativo, debe pagar una contribución especial al Fondo Nacional de Ciencia, Tecnología e Innovación la cantidad equivalente entre el cinco por ciento (5\%) y el diez por ciento (10\%) del valor de adquisición del software privativo. Este aporte debe efectuarse dentro del ejercicio fiscal correspondiente a la adquisición del programa.

\section{Conclusiones}

El análisis exegético de las disposiciones legales vigentes en el marco de las Políticas Públicas en Ciencia, Tecnología e Innovación, evidenciaron que el Estado venezolano, ciertamente ha establecido a través del andamiaje jurídico necesario, las bases estructurales de la organización del sistema nacional de ciencia tecnología e innovación, sobre las cuales se erige el puente comunicante entre la ciencia, la tecnología y su aplicación en la sociedad. Asimismo, se ha incorporado un conjunto de obligaciones tributarias, cuyo objetivo principal es aportar los recursos financieros necesarios para garantizar la gestión de la política pública, en forma autonómica con las características particulares que se detallan a continuación.

Queda sobre entendido que es la Locti, sin lugar a dudas el instrumento normativo que desarrolla el mandato constitucional; según el cual, en un primer orden, se establece la necesidad de articular una política pública que propulse la creación, organización, coordinación y financiamiento, del Sistema Nacional de Ciencia, Tecnología e Innovación. Así como la articulación de los órganos competentes de la formulación, seguimiento y evaluación de la política pública con el propósito firme de otorgarle sustentabilidad a la producción, la protección del ambiente, la seguridad y el ejercicio pleno de la soberanía nacional, acorde con la tendencia internacional.

Igualmente, se pudo constatar como el legislador nacional, con ese propósito, introduce instrumentos para su financiamiento mediante fondos recaudados por el mismo sistema, a través de la obligación parafiscal denominada aporte a la ciencia y la tecnología, el cual se genera, por la materialización del hecho imponible contemplado en la ley, y se cuantifica según la estimación de ingresos brutos en el año base en su última declaración ISLR; cuyo periodo de imposición cierra con el año calendario civil, y el pago durante los tres primeros meses del año siguiente.

En este orden de ideas, después de realizar una evaluación de racionalidad de la política de financiamiento de ciencia y tecnología en Venezuela, se puede afirmar que el diagnostico estructural refleja la institucionalización de una política pública propiamente dicha en ciencia y tecnología, toda vez, que ha sido dotada de un conjunto de órganos de la administración pública, de instrumentos de financiamiento autónomo, así como de una clara definición de las metas y estrategias, en coherencia con el mandato legal establecido, en el plan nacional para el desarrollo, lo que ciertamente lo califica como una política pública sostenible en inversión para el desarrollo $(i+d)$. 
No obstante, el desarrollo legislativo analizado evidencia un franco retroceso, específicamente en torno al ámbito subjetivo sobre el cual reposa la estructura organizativa del sistema, pues el texto de ley primigenio, aquella ley que creó al sistema de Ciencia y Tecnología en Venezuela (2005), concibió el supuesto de hecho, según el cual, las instituciones privadas dedicadas a la generación y desarrollo de productos científicos y tecnológicos, bajo la figura de aportante-beneficiario, podían invertir el pago de sus aportes, en la gestión y financiamiento de actividades propias de la empresa obligada, y/o en algún proyecto científico universitario de su preferencia, en virtud de las oportunidades que representaba para el mejoramiento productivo de su giro comercial, concertándose de esta forma, el vaso comunicante entre la ciencia, la tecnología y su aplicación directa en la sociedad, bajo un modelo de asociación estratégica de iniciativa privada, bajo la coordinación del órgano competente del sector público, y en coherencia con el diseño de la política púbica, procuraba la elevación del nivel de vida de la población, así como el impulso de la economía del país, fines extrafiscales de la administración financiera del Estado.

Sin embargo, esta viabilidad jurídica, resultó proscrita, toda vez que la naturaleza jurídica de esta obligación tributaria fue modificada, a partir de la reforma legislativa del año 2010, cuando en la disposición normativa que establece el ámbito subjetivo de aplicación de la ley (Art. 3), se puede observar una disminución cuantitativa en los numerales referidos, así como la eliminación de los sujetos privados del sistema nacional de ciencia y tecnología e innovación, en calidad de beneficiarios, pues, solo se les contempló como sujetos obligados al pago del aporte, lo que se traduce en la exclusión de la participación de la iniciativa privada directa, como sujetos propulsares de la ciencia y la tecnología, así como aliados estratégicos en el desarrollo y gestión de actividades científicos y tecnológicos, en el rol aportante-beneficiario.

Adicionalmente, en forma intempestiva se incorpora a las comunas como sujetos de derecho encargados de realizar e impulsar actividades de ciencia, tecnología, innovación y sus aplicaciones, en coherencia con la reforma de ley de la Administración Pública de (2008), que los incorporó como entes con competencias para el manejo y administración de recursos presupuestarios, entre los cuales se incluyeron los denominados Consejos Comunales y demás formas de organización comunitaria.

Lo que en definitiva, puede catalogarse como una desnaturalización de la esencia medular del sistema nacional de ciencia y tecnología, porque una cosa es la política pública dirigida a la promoción de la generación del conocimiento, la ciencia, la tecnología y la innovación, y otra arista de la ecuación, es la aplicación de los productos generados en el proceso científico en la resolución de los problemas de la sociedad, representada en este caso por los Consejos Comunales. Que en conjunción con la proscripción de la participación de la gestión privada en la aplicación de los aportes en beneficio mismo de los sujetos obligados por ley, desestimula la autogestión del conocimiento científico, que ciertamente representaba un estímulo para las grandes empresas obligadas al pago del aporte, quienes libremente podían decidir (durante la vigencia de la ley del 2005); entre mejorar la formación 
técnica y profesional de sus empleados, así como, la innovación de insumos y I o de procesos de producción, como mecanismo de cumplimiento voluntario de la prestación tributaria en especie, que involucraba el pago del aporte de ley, en el marco del espíritu, propósito y razón de legislador que creo la ley en el año 2005.

\section{Esta descontextualización} jurídica de la política, resultó mayormente profundizada, con la prohibición expresa de la reforma de ley del año 2014, del innovador mecanismo de financiamiento tributario parafiscal con fines extrafiscales, donde el legislador representado por el ejecutivo nacional, en ejercicio de la primera habilitación legislativa otorgada por la asamblea nacional, lo convirtió en un ordinario instrumento de recaudación de ingresos tributarios con fines fiscales, cuyo incumplimiento desencadena severas sanciones tributarias, y se faculta al organismo con competencia nacional, llámese Fonacit, a imponer sanciones pecuniarias especificadas en el texto de ley, y acentuadas con las sanciones tributarias contempladas en el Código Orgánico Tributario.

A estos aspectos sustantivos evidenciados, se le suma el conjunto de obligaciones tributarias contempladas en la ley del infogobierno, con la cual se pretende garantizar la aplicación de los adelantos de la tecnología de la innovación en la gestión de los asuntos del poder público nacional y los servicios que se prestan a la sociedad, todo ello, con financiamiento en los fondos provenientes de la recaudación de tributos parafiscales, cuyos sujetos pasivos son aquellos particulares del derecho privado, que se dediquen a la importación, distribución y comercialización de software privativo al Poder Público, y con otra obligación parafiscal, que recae sobre aquellos particulares que presten servicios de software privativos al Poder Público. Cuya alícuotas oscilan, entre dos punto cinco por ciento $(2,5 \%)$, de la utilidad neta del ejercicio, mientras que en el segundo caso lo será del uno punto cinco por ciento $(1,5 \%)$ de la utilidad neta del ejercicio, donde en ambos casos, las cantidades pagadas por concepto de estas nuevas contribuciones parafiscales serán deducibles del Impuesto Sobre la Renta.

Queda claro, que la institucionalización de la política pública en ciencia tecnología e innovación es impostergable, entendida como herramienta de gobierno que procura de la mejora de la calidad de vida de las personas, la gestión pública y de los servicios públicos, así como, el crecimiento de la producción interna del país, todo ello, con el fin último de fortalecer el desarrollo sostenible del país, y muy especialmente promoviendo vínculos entre educación, investigación y desarrollo, sin distinción entre publico y/o privado.

No obstante, el escenario normativo analizado, plantea la formulación de algunas reflexiones finales, referidas muy especialmente sobre la efectividad de la descontextualización del sistema de financiamiento que sufriere la política pública de ciencia tecnología e innovación experimentada en Venezuela, a propósito de las recurrentes reformas legislativas, las cuales han propiciado un distanciamiento entre la cadena de resultados originalmente propuestos, y que fungieron como presupuestos de hecho que propiciaron su diseño e incorporación como una política pública en el año 2005 y el contenido legislativo vigente.

\section{Referencias Bibliográficas}

\author{
Araque, Reyna, Sosa y Pittier (2006). "EI \\ Impacto Institucional De Las
}


Exacciones Parafiscales". VIII Jornadas Venezolanas de Derecho Tributario. Caracas, Venezuela.

Asamblea Nacional Constituyente (1999). "Constitución de la República Bolivariana de Venezuela". Gaceta Oficial de la República Bolivariana de Venezuela. № 36.860 .

Asamblea Nacional (2005). "Ley Orgánica De Ciencia, Tecnología E Innovación (Locti)". Gaceta Oficial No. 38.242 del 03 de Agosto de 2005.

Asamblea Nacional (2010). "Ley de Reforma de la Ley Orgánica de Ciencia, Tecnología e Innovación". Gaceta Oficial No 39.575 de fecha 16 de diciembre de 2010.

Asamblea Nacional (2013). "Ley del infogobierno". Gaceta Oficial No. 40.274 del 13 de octubre de 2013.

Clad (2013). "Carta Iberoamericana del Gobierno Electrónico". Aprobada por la IX Conferencia Iberoamericana de Ministros de Administración Pública y Reforma, 31 de mayo y $1^{\circ}$ de junio de 2007. Estado Pucón, Chile.

Ejecutivo Nacional (2014). "Decreto con fuerza y rango de ley de la Ley Orgánica de Ciencia, Tecnología e Innovación". Gaceta Oficial N ${ }^{\circ} 6.151$ Extraordinario de fecha 18 de noviembre de 2014.
Finol, Lorayne. (2009). Título del libro: El desarrollo institucional de la constitución de 1999 y los obstáculos para su plena vigencia. Capítulo del libro: LOCTI: INSTRUMENTO DE INTEGRACIÓN DE LA CIENCIA, ESTADO, Y SOCIEDAD. Editado: División de Extensión FCJP LUZ. Maracaibo, Estado Zulia. Venezuela.

Ejecutivo Nacional (2006). "Reglamento Parcial De La Ley Orgánica De Ciencia, Tecnología E Innovación". Decreto Presidencial No. 4891. Gaceta Oficial de la República Bolivariana de Venezuela No. 38.544 del 17/10/2006.

Musgrave, Richard. (1993). "Hacienda Pública Teórica y Aplicada". Quinta edición. Traductores: Juan Francisco Corona Ramón, Juan -Carlos Costas Terrones y Amelia Díaz Alvarez. Editorial Mc Graw Hill/Interamerican de España. Madrid España.

Subirats, Joan (2012). "Nuevos tiempos, ¿nuevas políticas públicas? Explorando caminos de respuesta". Publicado en la Revista del CLAD Reforma y Democracia. No. 54. Caracas. Venezuela.

Tribunal Supremo de Justicia. (2006). Sentencia de La Sala Político-Administrativa del Tribunal Supremo de Justicia. Consultada en: www.tsj.gov.ve. 


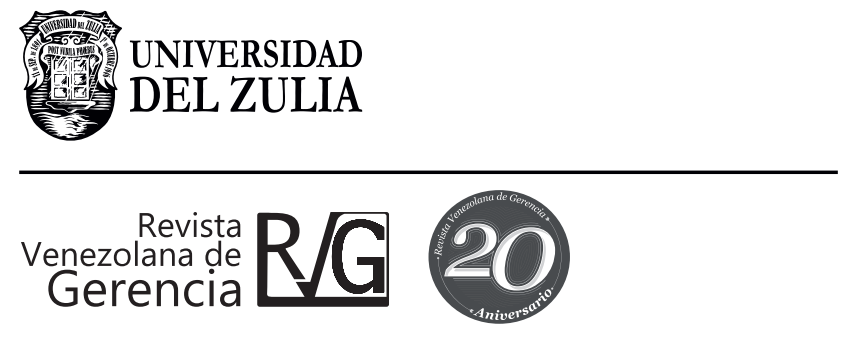

Año 21, No. 74

Esta revista fue editada en formato digital y publicada en junio del 2016, por la Universidad del Zulia, Vicerrectorado Académico, Serbiluz - Fondo editorial, Maracaibo -Venezuela. 